\title{
System Elements Model for Land Evaluation Information System
}

\author{
Mei Luo ${ }^{1, a}$ and Jianhong Jiang ${ }^{2, b}$ \\ ${ }^{1}$ College of management, Guilin University of Technology, Guilin Guangxi 541004, China \\ ${ }^{2}$ College of Business, Guilin University of Electronic Technology, Guilin, Guangxi 541004, China \\ aaubrey.luo@gmail.com, baero723@gmail.com
}

Keywords: Land evaluation information system; EBD; ROM; System elements

\begin{abstract}
This paper proposed a system elements model that can be used in LEIS system analysis phase before system development. The system elements model is constructed based on the EBD methodology. Recursive object model and question asking have been used to conduct a comprehensive environment analysis. LEIS system elements include stakeholder, data, documentation, function, performance, methodologies and cost. These elements restrict and interact with each other.
\end{abstract}

\section{Introduction}

As the constraints of land resource property and complexity of land resource management, land use issues not only have been very obvious at present with long term accumulation, but also have become increasingly prominent with the economic and social development [1]. For different purposes and different land type on the basis of the land evaluation, ultimately achieve the purpose of long-term sustainable development of the land. Information system, developing for land evaluation, would implement the automate evaluation, evaluation dynamic updates, and provide information and knowledge for the land evaluation phase, for this reason plenty of land evaluation information system (LEIS) have been developed.

In this paper, the LEIS system element is considered as a design problem and is analyzed from design point of view. Recursive object model (ROM) and question asking have been applied to such problems, and figured out the seven elements of LEIS eventually. The rest of the paper is organized as follows: next section includes analysis methodologies about EBD and ROM. Then an analysis process shows the implementation process of ROM and question asking. The work has been concluded at the end.

\section{Analysis Methodology}

In order to analyze the system elements of LEIS, the first part of Environment-Based Design (EBD) methodology is employed. Apart from traditional design methodologies, the EBD theory was logically derived from the axiomatic theory of design modeling, which was found on the recursive logic of design [2]. The basic idea is that a design problem is implied in a product system and is composed of three parts: the environment in which the designed product is expected to work, the requirements on product structure, and the requirements on product structure and performance are related to the product environment. In addition, the product environment includes three major environments: natural, built, and human. EBD includes three main steps: environment analysis, conflict identification, and solution generation. The objective of environment analysis is to find out the key environment components, in which the product works, and the relationships between the environment components, in which the product works, and the relationships between the environment components. From the environment implied in the design problem described by customer(s), the designer will introduce extra environment components that are relevant to the design problem at hands. The results from this analysis constitute an environment system. One of the key methods for environment analysis is linguistic analysis [2, 3]. The Recursive Object Model (ROM) was proposed by Zeng [4] to conduct this work. The ROM uses only five basic symbols to represent object, compound object, constraint 
relationship, predicate relationship and connection relationship. Those five elements are derived mathematically and are proven sufficient for technical English through enumeration. While a ROM diagram is generated, some questions should be asked to make every object in the ROM diagram clear. Wang and Zeng [5] gave the rules on question asking to conduct a comprehensive environment analysis. Two types of questions are asked in the process. One type of question, generated according to the topological structure of the ROM diagram, is domain - independent whereas the other relies on the domain of product development. A generic template is developed for generating the questions and for determining the sequence in which those questions are asked. The answers to the questions can be sought on the internet, in text books, the dictionary, the designer's own knowledge and experience, the customers and other partners involved in the product development, and/or nature itself. The generation of new questions may be based on the answers that are obtained. In order to verify the completeness of the extracted environment components and their relations, a roadmap was proposed as guidance for requirements modeling [6].

\section{System Elements Analysis}

For the system elements analysis, we started with one phrase: land evaluation information system. Based on Fig. 1, Environment analysis could be initialized by asking genetic questions. Rules on how to ask generic questions are defined in [5]. Some generic questions are asked: what is Information System? What is land evaluation? How to evaluate? Scholars who have studied information systems have different information system definition from different angles, this paper use the definition which Silver et al. [7] proposed: Use of information systems in an organization is to improve the organization of effectiveness and efficiency, the extent to which information systems can be organized by the ability of information systems environment, participatory and developers to achieve a common decision.

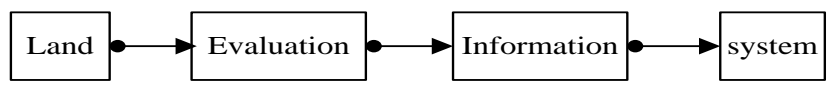

Figure 1. ROM diagram for the object

After those questions, the ROM diagram is updated with the answers as shown in Fig. 2. Following the same procedure, we can continue to ask questions regarding the unclear objects, the most updated ROM is shown in Fig. 3. It is easy to figure out the main LEIS objects which include stakeholder, data, documentation, function, performance, methodologies and cost.

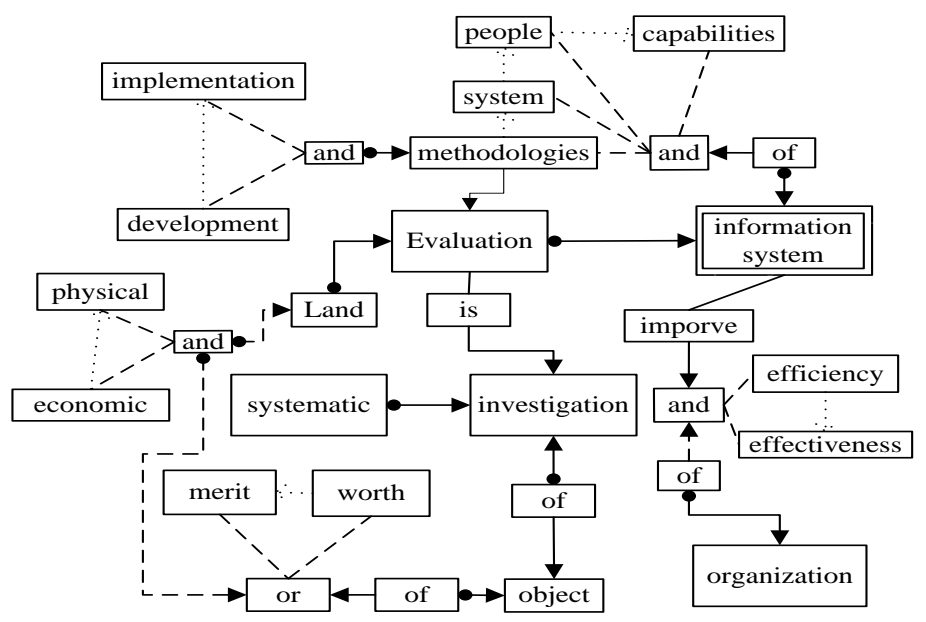

Figure 2. ROM diagram after the first generic question 


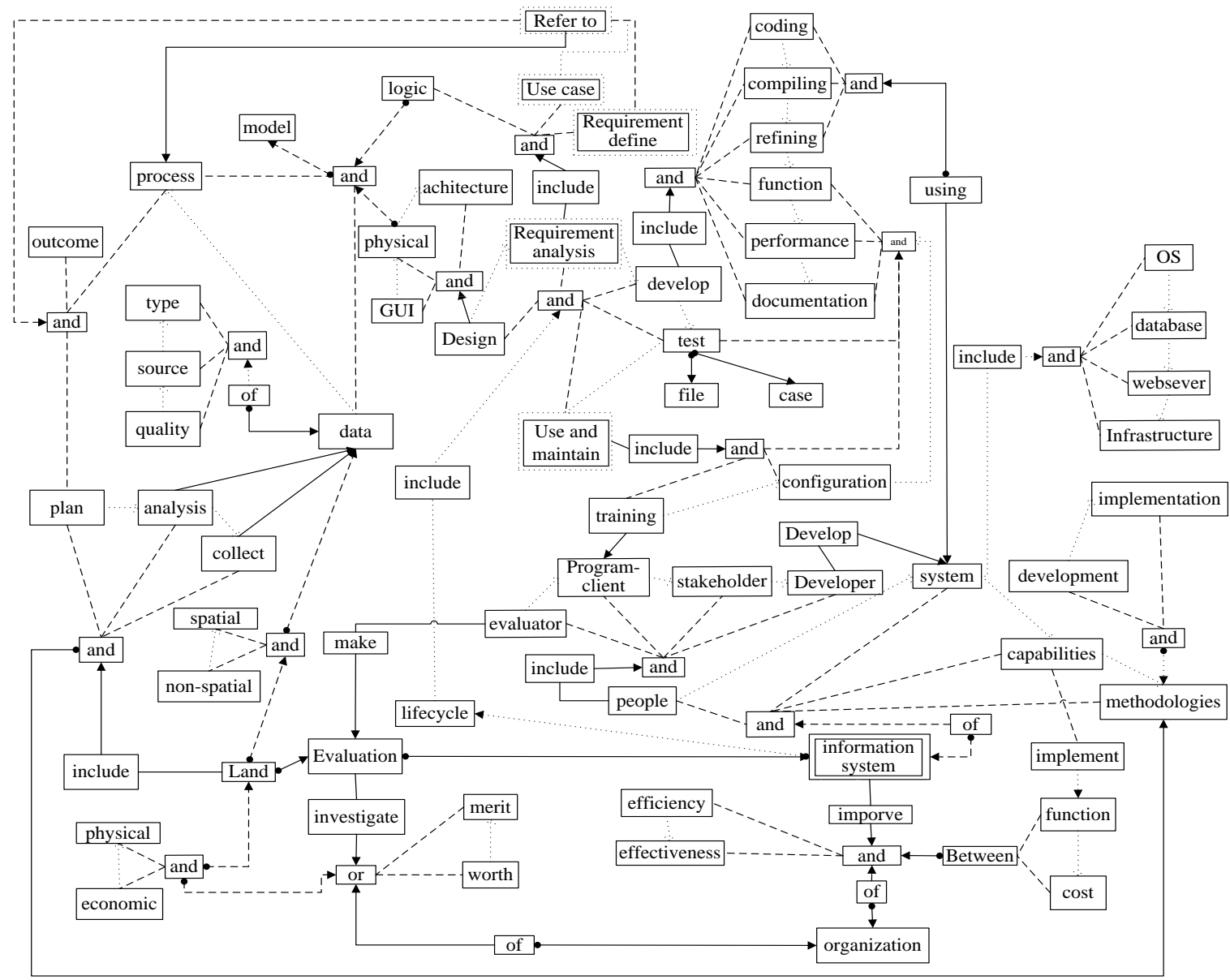

Figure 3. The most updated ROM diagram

The interaction and mutual restraint between these objects, Fig. 4 should be presented as the LEIS main elements relationship model. The LEIS structure could be formalized expressed as Eq. 1, where $\mathrm{Sh}$ is stakeholder, $\mathrm{C}$ is cost, $\mathrm{Da}$ is data, Dc is documentation, $\mathrm{F}$ is function, $\mathrm{P}$ is performance, $\mathrm{M}$ is methodologies.

$$
\oplus S=\oplus(S h \cup C \cup D a \bigcup D c \bigcup F \cup P \bigcup M)
$$

The significance and role of each element in the LEIS are:

Stakeholder refers to the organization or personal who actively participate in the system or whose interest could be affected by system execution and completion. In a board sense, stakeholder can be the specific executor as well as the internal and external related personnel of system development activities. In LEIS system development activities, the main stakeholder roles can be divided into the program clients, Evaluators and developers. Program clients is LEIS end users, since the LEIS features, Program client, include not only the internal and external users of the system, but also the system owner. Evaluators refer to evaluation client and implementation addition to the program client. Developers include systems analysts, system designers and system architects. In fact, system also includes other stakeholders who can benefit from system but not use system or interact with system directly. This kind of stakeholders do not involved in this article because they do not affect each other and have no direct relationship with LEIS elements.

Data is the fundamental purpose of information systems, is the symbol string such as text and numbers which can express the original fact occurs in the organization and its environment. Data is one of the organization's most important assets, representing the things of the objective world. Based on data mining, we can get a lot of valuable information, which is necessary for the organizational development. Data need to get some clues from its using environment to make it into information. There are three kinds of data including in LEIS development process, which are to build evaluation 
systems and evaluation models, to use by evaluation indicators, and to analysis. Data type is mainly composed of spatial and non-spatial data.

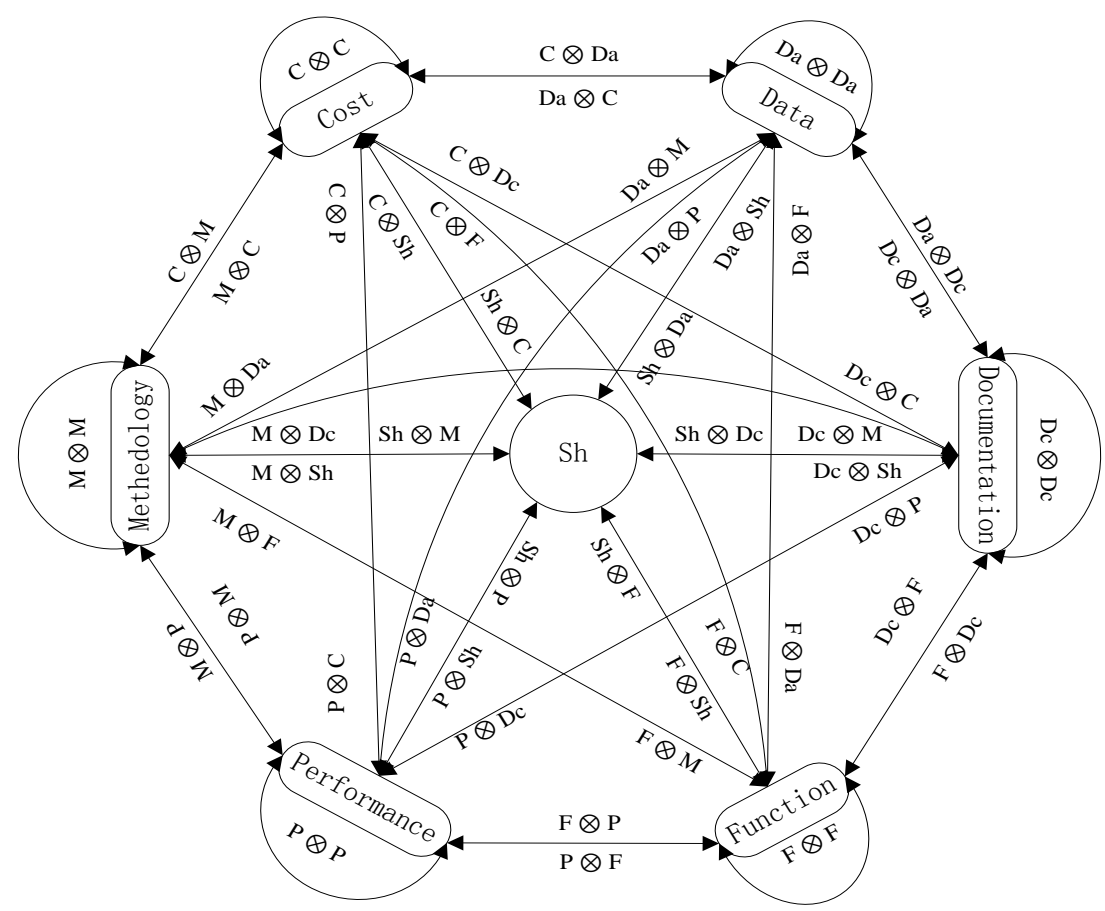

Figure 4. System elements model of LEIS

Distinguished as a data file, the information system document is the text data which mainly describe the system development and evolution process from scratch and each state. If there is no standardized system documentation or no system documentation, information system development, operation and maintenance will be in a turbid state, it will seriously affect the quality of the system, and even cause the failure of system development or operation [8]. The main documentation of LEIS is divided into two parts, one part is related to system development, use and maintenance, such as design documents, operating instructions, maintenance manuals, etc; the other part is related to the evaluation process and the evaluation results, each document is to analyze and summarize the information that support a particular function, such as evaluation reports, exception reports, the depth mining reports.

Most organizations are organized by function, and the system function is determined by the software [9]. As the organization-oriented system, LEIS have two main functions; the first one is to provide land-related decision information for management at different levels in the organization, and to provide land information for other system users to enable them to quickly identify opportunities and problems. The specific LEIS system function include input data collection, data storage, data transmission, data processing, document generation, query, and system management, etc.

System performance is mainly affected by the information technology, including hardware, software, network, the cumulative amount of data in the systems and information security. Using ' $3 \mathrm{~S}$ ' technology which are remote sensing, global position system and geographic information system to manipulate land-related data have a greater impact on system performance in LEIS, in addition to hardware, software, network providing support to information, decision and human-computer interaction.

Development and use of information systems involves a number of methodologies, the collection of methodologies affect the functions and performance of information system. There are two aspect methodologies involved in LEIS, evaluation methodologies and systems-related methodologies. Evaluation methodologies include indicator selection methodology, evaluation model determine methodology, questionnaire design and analysis methodology; system-related methodologies include 
system analysis, system design, system development methodology, database design methodology and data processing methodology.

Information system cost consists of three components: system development costs, operating costs and system maintenance costs. With the information system expanding range of applications in the organization and deepening the support for management functions [10], there are also information system growth costs. The costs in LEIS are divided into activity-based cost and functions achievement cost, activity-based cost associated with the evaluation activities, functions achievement cost associated with the system development. In order to maximize LEIS efficiency and effectiveness, we must effective control the work efficiency, schedule, personnel cost.

\section{Summary}

During the last few decades, LEIS have become very important for many governments and enterprise. However, instead of the pertinence analysis, LEIS system analysis phase is complex and diverse. For the work presented above, to help the client and developer, the major elements of LEIS were found out in a short time, the EBD theory is employed to resolve the LEIS system analysis problems from design point of view. With the powerful tool - ROM, and asking the right question, it become very easy to identify and clarify the main elements of the LEIS.

\section{Acknowledgements}

This research was supported by the Scientific Research Foundation of Guilin University of Technology (Grant No.002401003439), the Guangxi Natural Science Foundation (Grant No. 2014GXNSFCBA118287), and the Science and Technology program of Higher Education of Guangxi (KY2015LX139), all support is gratefully acknowledged.

\section{References}

[1] Meng J.J.: Land Evaluation and Management (CSPM, China 2011) (In Chinese)

[2] Chen, Z., Yao, S., Lin, J., Zeng, Y., and Eberlein, A.: International Journal of Manufacturing Research, Vol. 2 (2007) No. 3, p. 362.

[3] Chen, Z. "Formalization and classification of product requirements using axiomatic theory of design modeling" (MS., Concordia University, Canada, 2006).

[4] Zeng, Y.: Computer in Industry, Vol. 59 (2008) No. 6, p.612.

[5] Wang, M., and Zeng, Y.: international Journal of Computer Integrated Manufacturing, Vol. 22 (2009) No.4, p. 283.

[6] Chen, Z. Y., and Zeng, Y.: Concurrent Engineering, Vol. 14 (2006) No. 3, p. 219.

[7] Silver, M. S., Markus, M.L., and Beath, C.M.: MIS Quarterly, Vol. 19 (1995), No. 3, p. 361.

[8] Ralph M. Stair, George W. Reynolds: Principles of Information Systems: A Managerial Approach (Course Technology Press, United States 2003).

[9] Leonard Jessup, Joseph Valacich: Information Systems Today: Managing in the Digital World (Prentice Hall, United States 2007).

[10] Clemons E K, Dewan R M, Kauffman R J.: Journal of Management Information Systems, Vol. 21 (2004) NO. 2, p. 5. 